\title{
Essais
}

\section{La toilette mortuaire en soins palliatifs : une mise en corps et en espace de la mort}

The mortuary toilet in palliative care: Putting death into body and space

Frédérique Drillaud

\section{(2) OpenEdition}

Journals

Édition électronique

URL : https://journals.openedition.org/essais/8758

DOI : 10.4000/essais. 8758

ISSN : 2276-0970

Éditeur

École doctorale Montaigne Humanités

Édition imprimée

Date de publication : 15 avril 2021

Pagination : 97-102

ISBN : 979-10-97024-09-3

ISSN : 2417-4211

Référence électronique

Frédérique Drillaud, "La toilette mortuaire en soins palliatifs : une mise en corps et en espace de la mort », Essais [En ligne], 17 | 2021, mis en ligne le 27 avril 2021, consulté le 18 janvier 2023. URL : http://journals.openedition.org/essais/8758 ; DOl : https://doi.org/10.4000/essais.8758 


\section{La toilette mortuaire en soins palliatifs : une mise en corps et en espace de la mort}

Frédérique Drillaud

Dans les années 70 en France, un groupe d'experts a été mis en place afin de réfléchir à la prise en charge des malades en fin de vie et c'est en 1986, dans un contexte où le sida (maladie incurable) bouscule le monde médical, que les soins palliatifs ont été officialisés devenant alors un lieu institutionnel dédié à l'accompagnement des mourants. Ils représentent un milieu novateur au sein du modèle médical biotechnologique dominant, s'appuyant pour cela sur la théorie de la souffrance globale, "total pain ", élaborée dans les années 60 en Grande Bretagne par l'infirmière devenue médecin Cicely Saunders, une pionnière des soins palliatifs. Elle y recommande une approche holistique de la personne malade incluant pour cela l'accompagnement des dimensions physique, psychologique, sociale et spirituelle. En soins palliatifs, une équipe interdisciplinaire est présente afin d'accompagner la personne malade qui ne peut être guérie ainsi que son entourage. Dans ce contexte, la toilette mortuaire illustre à elle seule la large dimension symbolique et éthique que revêt l'accompagnement en soins palliatifs, lieu où la mort ne peut être évitée. Cet accompagnement ultime prodigué par les soignants, ce face-à-face avec la mort, correspond à une mise en corps et en espace de cette dernière où sont aussi bien considérés les vivants que les morts.

À l'aide de mes différents terrains anthropologiques, j'illustrerai dans un premier temps la démarche des soins palliatifs à travers le récit d'une toilette mortuaire, et dans un second temps, il s'agira d'explorer ce qui se joue lorsque la mort est prise en considération.

\section{La toilette mortuaire d'Anna}

Anna a 90 ans, elle est d'origine autrichienne et a pour langue maternelle l'allemand. Elle a appris le français au Liban lorsqu'elle a rencontré un italien parlant français, qui est devenu son mari. De leur union sont nés deux garçons qui ont aujourd'hui 69 et 65 ans. L'ainé, qui a deux fils également, vit dans la région bordelaise, son frère quant à lui vit en Bretagne et n'a pas d'enfant. 
Anna est veuve d'une seconde union et vit depuis avec une amie de longue date dans une maison en région bordelaise. En provenance du service de gériatrie, elle a été accueillie dans le service de soins palliatifs le 14 décembre suite à un choc septique (une défaillance circulatoire aiguë). Elle y décèdera le 18 décembre. Ce matin-là, il était prévu que j'assiste à l'entretien d'accueil d'une personne arrivée dans le service, mais la cadre de santé m'annonça qu'Anna venait de décéder et que je pouvais assister à sa toilette mortuaire si je le souhaitais toujours. En effet, ce même matin, je lui avais demandé s'il serait possible, si les soignants étaient d'accord, que je puisse assister à une toilette mortuaire. Il me semblait en effet important d'assister aux différentes pratiques de l'accompagnement en soins palliatifs mais cette dernière revêtait un caractère particulier. En effet, les pratiques auxquelles j'avais assisté jusquelà étaient en lien avec des personnes mourantes certes, mais encore vivantes et conscientes de ma présence. Dans le cas de la toilette mortuaire, ce ne serait pas le cas et bien qu'il fût évident pour moi d'y assister si cela était possible afin de mieux comprendre ce qui se joue jusqu'au dernier soin, je ressentis également une forme d'imposture à vivre ce moment. Imposture qui s'est dissipée lorsque j'ai su qu'une aide-soignante stagiaire serait présente également et lorsque j'ai pu ressentir l'accueil positif qui était fait à ma demande. J'allais avoir l'occasion et l'honneur d'assister non seulement à ce soin ultime mais également à la transmission de cette pratique entre soignants.

Je n'avais pas eu l'occasion de rencontrer Anna auparavant, la seule connaissance que j'avais d'elle était la fiche synthèse réalisée par le médecin. Lorsque l'aide-soignante stagiaire et moi-même sommes entrées dans la chambre, une infirmière et une aide-soignante étaient en train de préparer la chambre en vue de cette toilette, le corps d'Anna reposait sur le lit. Nous nous sommes installées spontanément dans un coin afin de perturber le moins possible ce qui allait se produire sous nos yeux. Je n'imaginais pas qu'à travers les gestes et les paroles des soignantes je ferai quelque part un peu connaissance avec Anna. En effet, une musique classique les accompagnait, celle quadorait Anna comme nous dit l'infirmière. Elle nous montre alors un bracelet sur lequel doit être indiqué le nom, la date et l'heure du décès pour le dépositoire et précise que pour sa part, elle attend toujours que les proches soient passés dire un dernier au revoir avant de poser le bracelet au poignet. Je pense alors à ce parallèle frappant avec la naissance, à la différence près qu'en maternité, les parents repartent avec le bracelet de naissance de leur enfant tel un trophée.

Nous assistons alors à la mise en ordre de la chambre qui consiste à démédicaliser l'espace en enlevant tous les objets techniques et à mettre en avant les objets intimes d'Anna. Les cartes et photos de ses proches sont disposées harmonieusement sur une table auprès d'elle, avec son parfum et une orchidée à laquelle elle tenait. L'infirmière nous dit que lorsque la personne est croyante, en indiquant que ce n'est pas le cas d'Anna et que c'est d'ailleurs de moins en 
moins le cas, mais lorsque ça l'est et que la personne a un objet de culte, il est déposé sur son lit. Elle nous explique que cette mise en place est réalisée afin de faciliter l'accessibilité des proches, le lien, et que ce n'est pas facile. À partir de ce moment-là, la parole est réservée à Anna à qui l'infirmière s'adresse comme si elle était encore vivante. Alors qu'elle prévient Anna des gestes délicats comme l'obstruction des orifices naturels, l'aide-soignante lui tient la main. Il en sera de même peu après lorsque le médecin passera pour effectuer l'ablation du pacemaker d'Anna, acte obligatoire. Cette parole adressée à un corps sans vie semble aller de soi pour ces trois soignantes. Une fois ce geste réalisé, le médecin caresse Anna et laisse les soignantes œuvrer. C'est ensuite une alternance de gestes techniques et de gestes personnalisés, tous effectués avec une extrême délicatesse aussi bien par l'infirmière que par l'aide-soignante. La même délicatesse que j'ai pu relever lors de mes observations précédentes, à la seule différence que les personnes étaient encore vivantes. L'interaction entre ces soignantes ne passe alors plus par la parole mais uniquement par des regards complices qui rythment ces gestes. La toilette est réalisée de haut en bas du corps, Anna est ensuite habillée avec des vêtements qu'elle affectionnait, coiffée avec sa brosse aspergée de son parfum et tout en lui mettant du baume sur les lèvres, l'infirmière lui dit " J'ai acheté le même baume à lèvres que vous. "

Après plus d'une heure de soin, nous nous retirons en laissant derrière nous le corps sans vie d'Anna dans un espace organisé de manière à ce que ses proches puissent s'y retrouver. Dans le couloir, l'aide-soignante stagiaire évoque le fait que les toilettes mortuaires se font de moins en moins, à quoi l'infirmière répond : "Ici, le soin ne s'arrête pas à 11 h05 lorsque le cour a cessé de battre ».

Elle lui explique alors qu'elle a pu se libérer une heure, ce qui n'existe pas dans d'autres services, parce qu'en soins palliatifs c'est un soin au même titre que les autres, et elle lui rappelle qu'ils ont pour mission d'accompagner non seulement les personnes malades mais aussi leurs proches et qu'à travers ce dernier soin c'est aussi l'accompagnement des proches qui est réalisé.

En effet, à travers la toilette mortuaire, au-delà des gestes techniques, le soignant effectue des gestes symboliques, respectueux de la singularité du patient et de son entourage qu'il a accompagné jusque-là. La chambre hospitalière devient le lieu d'une ritualisation qui semble mettre en scène, à l'aide de mots, de gestes et de silences, le passage de la présence à l'absence de l'occupant de cette chambre ainsi que le passage du visible à l'invisible de l'occupant de ce corps. À travers la matérialisation de la préparation du corps du défunt et de l'espace chambre semble se jouer la configuration d'un espace imaginaire lié à la mort où le prendre soin ne s'arrête pas lorsque le cœur a cessé de battre. Ce dernier soin semble être l'aboutissement de tous les soins qui l'ont précédé, il y règne le même respect du lien à l'autre qui sous-tend la démarche palliative. 
À travers la toilette mortuaire, les gestes et les paroles de ces soignantes en lien à ce corps sans vie témoignent du lien aux autres vivants. Aussi, cela soulève la question suivante : à travers le face-à-face avec la mort, est-ce un face-à-face avec la vie qui se révèle alors ?

\section{L'effet-miroir de la mort sur la vie}

La fin de vie en soins palliatifs est un contexte tout à fait spécifique qui soulève de nombreuses questions dans le sens où la mort n'est pas encore, mais l'issue vers elle est certaine.

On peut alors se demander quelle expérience font la personne et les personnes qui l'accompagnent sur ce chemin.

Lobservation participante en tant que bénévole accompagnante en fin de vie que j'ai réalisée en institution et à domicile durant deux ans et le travail de recherche qui en découla, ont montré que la personne malade confrontée à sa mort proche est alors en quête de sens, ce qui la mène à une dimension réflexive au regard de sa vie passée. Les questions émergentes semblent s'inscrire dans son lien à l'autre, et son besoin de reconnaissance en tant que personne et non en tant que malade devient alors central. Ce questionnement semble s'opérer sous l'effet-miroir de la mort sur la vie qui conduit la personne concernée à une prise de conscience de l'importance de la relation à l'autre. Effet-miroir s'entendant ici comme la transmission d'un reflet dont le contenu modifie le sujet regardant. Lors de mes accompagnements, il était courant que les personnes accompagnées racontent leur vie, me plaçant alors comme témoin de leur parcours de vie. L'expression de l'expérience vécue était pour ces personnes une manière de s'inscrire dans la vie sociale et d'assurer ainsi une certaine permanence de leur identité à travers la reconnaissance d'une personne témoin. C'est à travers le récit de sa vie que la personne confrontée à la proximité de sa mort permet sa permanence et en vient à transmettre ce que le philosophe Paul Ricœur ${ }^{1}$ appelle «l'identité narrative ». À ses yeux, une même racine irrigue l'histoire et la mémoire : le récit. Et c'est à travers lui, par un mode de conscience de soi, qu'on en vient à l'identité. Ce qui explique que depuis quelques années des biographes hospitaliers interviennent dans certains services de soins palliatifs, créant ainsi un nouveau type d'accompagnement.

Une autre étude de terrain menée au sein d'une Unité de Soins Palliatifs a laissé apparaître ce même effet-miroir mais cette fois sous l'angle des soignants. La proximité avec la mort semble donner un sens à leur vie qui se traduirait à travers le lien à l'autre et la volonté de prendre soin de cet autre. Tous les professionnels entretenus ne sont pas dans ce service par hasard et ont du mal à s'imaginer travailler ailleurs. Et beaucoup de ceux que j'ai rencontrés par

1 Paul Ricœur, La mémoire, l'histoire, l'oubli, Paris, Le Seuil, 2000. 
ailleurs, y compris au Canada, disent la même chose. Ces soignants n'ont pas manqué de relever le phénomène qui conduit les personnes à l'approche de la mort à une prise de conscience de ce qui a vraiment de la valeur à leurs yeux au regard de leur vie et d'ainsi donner un sens à leur parcours de vie.

L'anthropologue Yannis Papadaniel décrit la manière dont certains bénévoles d'accompagnement en Suisse tirent un enseignement de leur pratique pour leur propre vie, ces derniers parlant même de " la beauté de la mort " : "Leur expérience de la fin de vie leur donnerait accès à un essentiel, en les enjoignant de ne se focaliser que sur ce qui a vraiment de la valeur ${ }^{2}$, ce que j'ai observé également sur le terrain français. J'ai pu en effet constater que les membres de l'équipe accompagnante n'étaient pas exempts de cet apprentissage, un processus de "don contre don "s'opérant entre eux et les personnes malades. Le philosophe Frédéric Worms ${ }^{3}$ parle pour cela d'une expérience de rencontre où deux personnes adviennent à elles-mêmes. Il considère que la situation palliative révèlerait l'importance du soin dans nos vies mais également dans notre société, parlant pour cela de priorité.

L'effet-miroir de la mort sur la vie révèle l'importance du lien à l'autre qui s'exprime alors au travers de différentes attentions de la part de l'équipe soignante envers les personnes malades et leurs proches. Outre l'accompagnement institutionnel, la considération de l'autre peut également passer par un chant improvisé et spontané par un binôme infirmière/aide-soignante dans la chambre d'une patiente qui est sensible à cela, l'organisation d'un apéritif dans la chambre d'un patient (une cave à vin est présente dans le service), dans des cadeaux de Noël personnalisés à l'aide des informations récoltées sur chaque patient par chaque membre de l'équipe interdisciplinaire...

Ce qui pourrait ressembler à des détails, mais des détails qui permettent à une soignante de dire : "Ce que j'aime ici, c'est qu'on porte les lunettes humanités ».

Un espace dédié où la mort n'est pas occultée mais accompagnée semble révéler la leçon de la mort sur la vie, mettant en exergue les notions de quête de sens et de reconnaissance où la relation à l'autre devient primordiale. La toilette mortuaire en soins palliatifs révèle que le soin porté aux morts met également en jeu le soin des vivants. Comme en témoignent les travaux des sociologues Louis-Vincent Thomas ${ }^{4}$ et Patrick Baudry ${ }^{5}$, la mort se situe à l'articulation du singulier et du collectif et questionne les dimensions individuelles et collectives de la société.

2 Yannis Papadaniel, La mort à côté, Toulouse, Anacharsis, 2013, p. 30.

3 Frédéric Worms, Le moment du soin. À quoi tenons-nous?, Paris, PUF, 2010.

4 Louis-Vincent Thomas, Anthropologie de la mort, Paris, Payot, 1975.

5 Patrick Baudry, La place des morts. Enjeux et rites, Paris, L'Harmattan, 2006 (1999). 


\title{
À travers l'effet-miroir de la mort sur la vie, ce sont les formes de solidarité et d'organisation de notre société qui sont interrogées.
}

\author{
Frédérique Drillaud \\ MICA EA 4426 \\ Université Bordeaux Montaigne \\ f.drillaud@sfr.fr
}

\begin{abstract}
Résumé
En soins palliatifs, une équipe interdisciplinaire est présente afin d'accompagner la personne malade qui ne peut être guérie ainsi que son entourage. Dans ce contexte, la toilette mortuaire illustre à elle seule la large dimension symbolique et éthique que revêt l'accompagnement en soins palliatifs, lieu où la mort ne peut être évitée. Cet accompagnement ultime prodigué par les soignants, ce face-à-face avec la mort, correspond à une mise en corps et en espace de cette dernière où sont aussi bien considérés les vivants que les morts. La chambre hospitalière devient le lieu d'une ritualisation qui semble mettre en scène, à l'aide de mots, de gestes et de silences, le passage de la présence à l'absence de l'occupant de cette chambre. À travers la matérialisation de la préparation du corps du défunt et de l'espace chambre semble se jouer la configuration d'un espace imaginaire lié à la mort où le prendre soin ne s'arrête pas lorsque le cœur a cessé de battre. Un espace dédié où la mort n'est pas occultée mais accompagnée semble révéler la leçon de la mort sur la vie, mettant en exergue les notions de quête de sens et de reconnaissance où la relation à l'autre devient primordiale. À travers l'effet-miroir de la mort sur la vie, ce sont les formes de solidarité et d'organisation de notre société qui sont interrogées.
\end{abstract}

\section{Mots-clés}

Toilette mortuaire, soins palliatifs, mort, vie, lien.

\begin{abstract}
In palliative care, an interdisciplinary team is present to accompany the sick person who cannot be cured and their family and friends. In this context, the mortuary toilet alone illustrates the broad symbolic and ethical dimension of palliative care accompaniment, a place where death cannot be avoided. This ultimate accompaniment provided by the caregivers, this face-to-face with death, corresponds to a setting in body and space of the latter where both the living and the dead are considered. The hospital room becomes the site of a ritual that seems to stage, with the help of words, gestures and silences, the passage from the presence to the absence of the occupant of this room. Through the materialization of the preparation of the body of the deceased and the bedroom space seems to play out the configuration of an imaginary space linked to death. A dedicated space where death is not occulted but accompanied seems to reveal the lesson of death over life, highlighting the notions of the quest for meaning and recognition where the relationship with the other becomes paramount. Through the mirror effect of death on life, the forms of solidarity and organization of our society are questioned.
\end{abstract}

\section{Keywords}

Mortuary toilet, palliative care, death, life, connection. 